\title{
Acute disseminated encephalomyelitis with bilateral optic neuritis following ChAdOx1 COVID-19 vaccination
}

\author{
Sai A. Nagaratnam ${ }^{1 *}$, Alex C. Ferdi ${ }^{2}$, John Leaney ${ }^{3}$, Raymond Lam Kwong Lee ${ }^{4}$, Yun Tae Hwang ${ }^{5}$ and \\ Robert Heard ${ }^{6}$
}

\begin{abstract}
Background: Acute disseminated encephalomyelitis (ADEM) is a rare immune-mediated inflammatory demyelinating disease of the central nervous system. We report a case of ADEM presenting with bilateral optic neuritis temporally associated with the ChAdOX1 vaccine against SARS-COVID19 virus.

Case presentation: A 36-year-old female presented with bilateral optic neuritis following her first dose of the ChAdOx1 vaccine. Initial MRI Brain showed evidence of demyelination within the subcortical white matter, with no radiological involvement of the optic nerves. Visual evoked potentials were consistent with bilateral optic neuritis which was confirmed radiologically on follow up MRI. She was treated with intravenous steroids with improvement both in symptoms and radiological appearance. A pseudo-relapse occurred which was treated with a further course of intravenous steroids followed by an oral taper. The clinical, radiological and serological results were most consistent with diagnosis of ADEM.
\end{abstract}

Conclusions: ADEM is an exceedingly rare complication of $\mathrm{ChAdOx} 1$ vaccine despite millions of doses. While it is imperative clinicians remain aware of neurological complications of vaccines, the importance of vaccination to control a pandemic should not be undermined.

Keywords: Acute disseminated encephalomyelitis, Oxford Astra Zeneca coronavirus disease 2019 vaccine, Severe acute respiratory syndrome coronavirus 2, Demyelination, Case report

\section{Background}

Acute disseminated encephalomyelitis (ADEM) is an immune-mediated demyelinating disease of the central nervous system [1]. It is a rare entity, with an estimated annual incidence of 0.23 to 0.4 per 100,000 children and 0.26 per 100,000 adults [2, 3]. ADEM often occurs following an antigenic challenge such as a viral illness or vaccination although it is considered unusual following vaccination, and only $5 \%$ of patients with ADEM are

\footnotetext{
*Correspondence: sai@nagaratnam.net

1 Department of Neurology, Gosford Hospital, Gosford, NSW, Australia Full list of author information is available at the end of the article
}

estimated to have received a vaccine during the preceding month [4].

The virus responsible for the current global pandemic, SARS-CoV-2 infection has been associated with ADEM as well [5]. Since the vaccination efforts to combat the pandemic began, there have also been number of published reports of possible ADEM following SARS-CoV-2 vaccination: three related to ChAdOx1 (AstraZeneca, Oxford, United Kingdom), a non-replicating viral vector vaccine; two related to Sinovac (Vero Cells, Beijing Institute of Biological Products Co., Ltd., Beijing, China), an inactivated SARS-CoV-2 vaccine, and one following Cominarty (Pfizer-BioMTech, New York, New York, 
USA), an mRNA vaccine [6-11]. There have been more cases of ADEM possibly related to ChAdOx1 vaccines that have been reported to authorities. Here, we present a case of probable ADEM presenting with bilateral optic neuritis occurring following administration of the ChAdOx1 vaccine.

\section{Case presentation}

A 36-year-old woman presented to her local emergency department with a 2 day history of a right sided headache, photophobia and blurred vision in the right eye, 14 days after her first dose of ChAdOx1 vaccine. At this time, the neurological examination, including visual acuity, performed in the emergency department was reportedly normal. Her D-dimer and platelet count were $0.74 \mathrm{mg} / \mathrm{L}$ $(<0.5 \mathrm{mg} / \mathrm{L})$ and $258 \times 10^{9} / \mathrm{L}\left(150-400 \times 10^{9} / \mathrm{L}\right)$ respectively. CT venography showed no evidence of venous sinus thrombosis and thrombosis with thrombocytopenia syndrome was considered unlikely.

She returned to the emergency department 2 days later with bilateral visual impairment and subjective colour desaturation, painful eye movements and fatigue. She did not report any weakness, sensory disturbances or bowel or bladder incontinence. Visual acuity was $6 / 15(-2)$ on the right and $6 / 30$ on the left which did not correct with pinhole. There was no relative afferent pupillary defect or asymmetric red desaturation. There was full range of eye movements without nystagmus or diplopia, although the eye movements themselves were painful. The rest of the upper and lower limb examination was unremarkable. She was admitted to the hospital on this occasion.

She had no significant personal medical history, including previous episodes of weakness or sensory changes and was not a migraineur. She had no known contact with COVID-19 positive cases, and no respiratory symptoms, hence a SARS-CoV-2 polymerase chain reaction test was not performed, as per the local testing protocol at that time. Her family history was notable for mother with multiple sclerosis and the patient had a normal MRI brain 5 years prior, prompted by her mother's diagnosis.

Biochemical analysis, summarised in Table 1, did not reveal any significant abnormalities.

Cerebrospinal fluid analysis performed on day 2 of admission showed a normal protein of $0.4 \mathrm{~g} / \mathrm{L}(0.19-$ $0.56 \mathrm{~g} / \mathrm{L})$, glucose of $4.8 \mathrm{mmol} / \mathrm{L}(2.8-4.5 \mathrm{mmol} / \mathrm{L})$ with pleocytosis (white cell count $\left.59 \times 10^{6} / \mathrm{L}\right)\left(<5 \times 10^{6} / \mathrm{L}\right)$. CSF IgG was $0.06 \mathrm{~g} / \mathrm{L}(<0.03 \mathrm{~g} / \mathrm{L})$ with serum IgG of $12.4 \mathrm{~g} / \mathrm{L}(7.0-16 \mathrm{~g} / \mathrm{L})$, and oligoclonal IgG bands were present, suggestive of intrathecal IgG synthesis. Serum and CSF aquaporin 4 antibodies were negative. Serum myelin oligodendrocyte glycoprotein antibody (MOG) was negative. Visual evoked potentials performed on Day

Table 1 Biochemical and serological results

\begin{tabular}{|c|c|c|}
\hline Biochemistry and serology results & Result & Normal Range \\
\hline Platelet count & $225 \times 10^{9} / L$ & $150-400 \times 10^{9} / \mathrm{L}$ \\
\hline Vitamin D & $60 \mathrm{nmol} / \mathrm{L}$ & $50-140 \mathrm{nmol} / \mathrm{L}$ \\
\hline Folate & $29.1 \mathrm{nmol} / \mathrm{L}$ & $7-46.4 \mathrm{nmol} / \mathrm{L}$ \\
\hline Vitamin B12 & $309 \mathrm{pmol} / \mathrm{L}$ & 138- $652 \mathrm{pmol} / \mathrm{L}$ \\
\hline Beta 2 glycoprotein antibodies & $6 \mathrm{U} / \mathrm{ml}$ & $<20 \mathrm{U} / \mathrm{ml}$ \\
\hline Cardiolipin lgG & $<3 \mathrm{U} / \mathrm{ml}$ & $<20 \mathrm{U} / \mathrm{ml}$ \\
\hline Cardiolipin 1gM & $2 \mathrm{U} / \mathrm{ml}$ & $<20 \mathrm{U} / \mathrm{ml}$ \\
\hline Anti nuclear antibodies & Not detected & \\
\hline Extractable nuclear antibodies & Not detected & \\
\hline Anti-neutrophil cytoplasmic antibodies & Not detected & \\
\hline Serum aquaporin 4 antibodies & Negative & \\
\hline CSF aquaporin 4 antibodies & Negative & \\
\hline Serum myelin oligodendrocyte glycoprotein antibody & Negative & \\
\hline $\begin{array}{l}\text { CSF PCR testing - N meningitidis DNA, S pneumoniae DNA, Herpes simplex virus type } 1 \text { DNA, Herpes } \\
\text { simplex virus type } 2 \text { DNA, Human parechovirus RNA, VZV DNA, Enterovirus RNA }\end{array}$ & Not detected & \\
\hline CSF Glucose & $4.8 \mathrm{mmol} / \mathrm{L}$ & $2.8-4.5 \mathrm{mmol} / \mathrm{L}$ \\
\hline CSF Protein & $0.4 \mathrm{~g} / \mathrm{L}$ & $0.19-0.56 \mathrm{~g} / \mathrm{L}$ \\
\hline CSF White cells & $59 \times 10^{6} / \mathrm{L}(99 \%$ mononuclear) & $<5 \times 10^{6} / \mathrm{L}$ \\
\hline CSF Red cells & $3 \times 10^{6} / \mathrm{L}$ & $<1 \times 10^{6} / \mathrm{L}$ \\
\hline CSF culture & Negative & \\
\hline CSFIgG & $0.06 \mathrm{~g} / \mathrm{L}$ & $<0.03 \mathrm{~g} / \mathrm{L}$ \\
\hline Serum lg $G$ & $12.4 \mathrm{~g} / \mathrm{L}$ & $7-16 \mathrm{~g} / \mathrm{L}$ \\
\hline
\end{tabular}




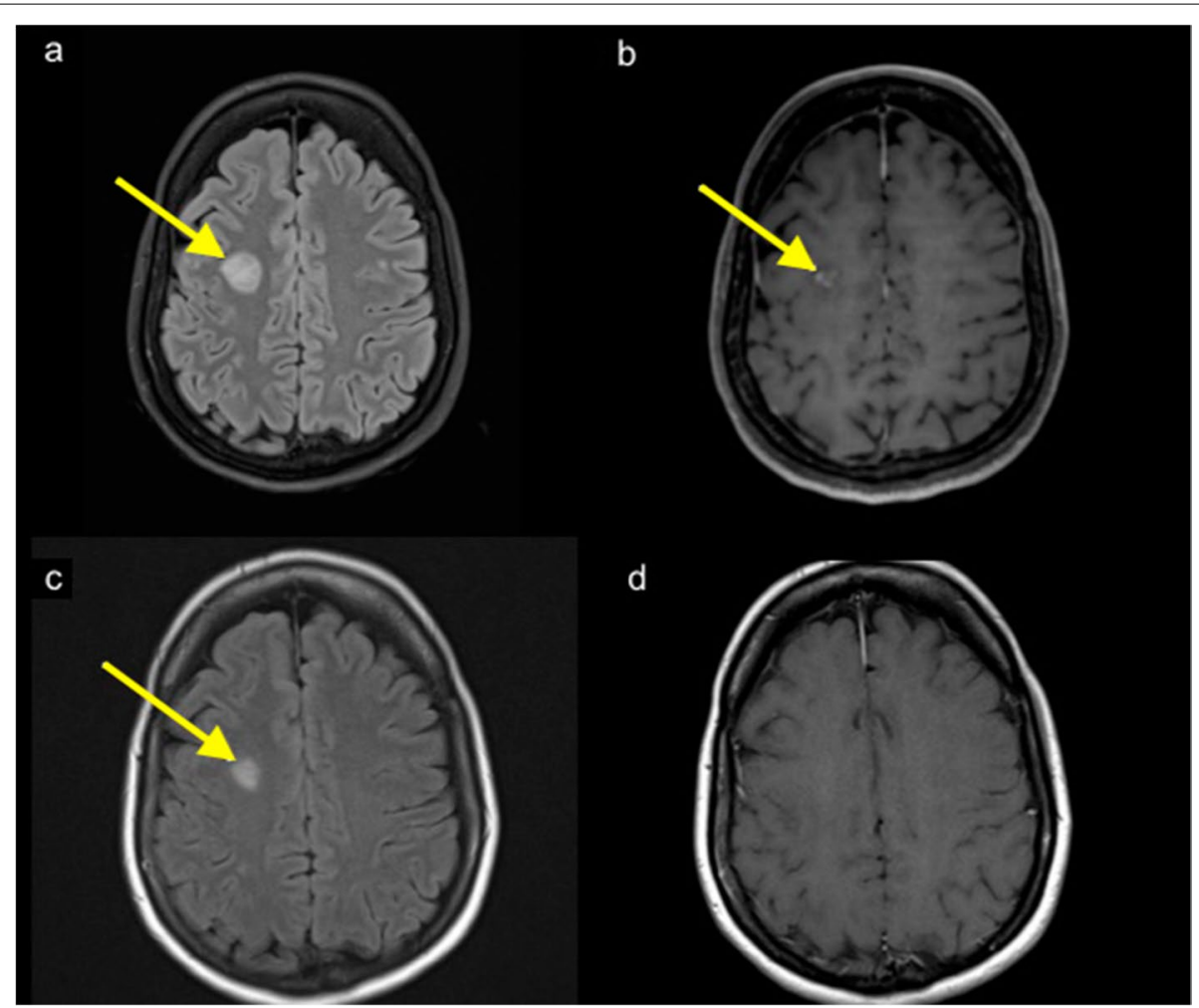

Fig. 1 MRI Brain. a. Hyperintense FLAIR signal white matter lesion in the right frontal lobe (arrow). b Internal punctate enhancement of the right frontal lobe lesion (arrow). c Lesion shown in Fig. 1a reduced in size after 2 weeks (arrow). $\mathbf{d}$ Lesion no longer demonstrated abnormal enhancement

1 were unrecordable from the left eye and delayed on the right, consistent with demyelinating pathology involving the anterior visual pathways bilaterally but more severe on the left.

3 T MRI brain (Skyra, Siemens, Germany) also performed on Day 2 showed multiple T2/ FLAIR hyperintense lesions involving the subcortical white matter, posterior limb of bilateral internal capsules, pons and left middle cerebellar peduncle. The largest lesion was in the right frontal centrum semiovale measuring $17 \times 17 \mathrm{~mm}$ with multiple internal punctate foci of gadolinium contrast enhancement (Fig. 1a, b). There was no callosal involvement. Notably, there was no definite abnormal signal or enhancement of optic nerves. MRI of the spine showed no evidence of demyelinating disease.

Ophthalmology examination on Day 8 found visual acuity of $6 / 5(20 / 16)$ in the right eye and 6/9 (20/30) in the left. Colour vision was depressed in both eyes with 12/17 Ishihara plates identifiable with the right eye and $10 / 17$ with the left. There was no relative afferent pupillary defect detectable. The patient's optic discs appeared healthy with no swelling or atrophy (Fig. 2). There was no concurrent anterior segment or retinal disease. Formal visual fields were assessed with Humphrey Field Analyzer (Carl Zeiss Meditec, Dublin, CA, USA), which demonstrated largely intact fields in both eyes, with only a few mild scattered non-specific deficits (Supplementary file 1). Optical coherence tomography (CIRRUS ${ }^{\mathrm{TM}}$ HD-OCT 5000, Carl Zeiss Meditec, Dublin, CA, USA) showed mild swelling of her retinal nerve fibre layer bilaterally, measuring a total thickness of $111 \mu \mathrm{m}$ on the right and $108 \mu \mathrm{m}$ on the left (Supplementary file 2).

Based on these results, we concluded that the diagnosis that would best fit the clinical scenario and the investigation results was ADEM presenting with optic neuritis. She received 3 daily doses of $1000 \mathrm{mg}$ of intravenous methylprednisone with marked improvement in vision and discharged home with plans for close outpatient follow up but without tapering regime of oral steroids.

On day 15, the patient reported worsening of vision in both eyes, worse in the left. Examination revealed further reduction in visual acuity in the left eye of $6 / 180(20 / 200)$ and subtle reduction in the right eye of $6 / 6(20 / 20)$. The colour vision was reduced to $0 / 17$ Ishihara plates in the right eye and could not be assessed in the left. A dense relative afferent pupillary defect was present in the left eye. Optic nerves remained normal clinically with no swelling or atrophy. Loss of visual acuity in the left eye resulted in a sensory exotropia with poor fixation that precluded visual field or optical coherence tomography 


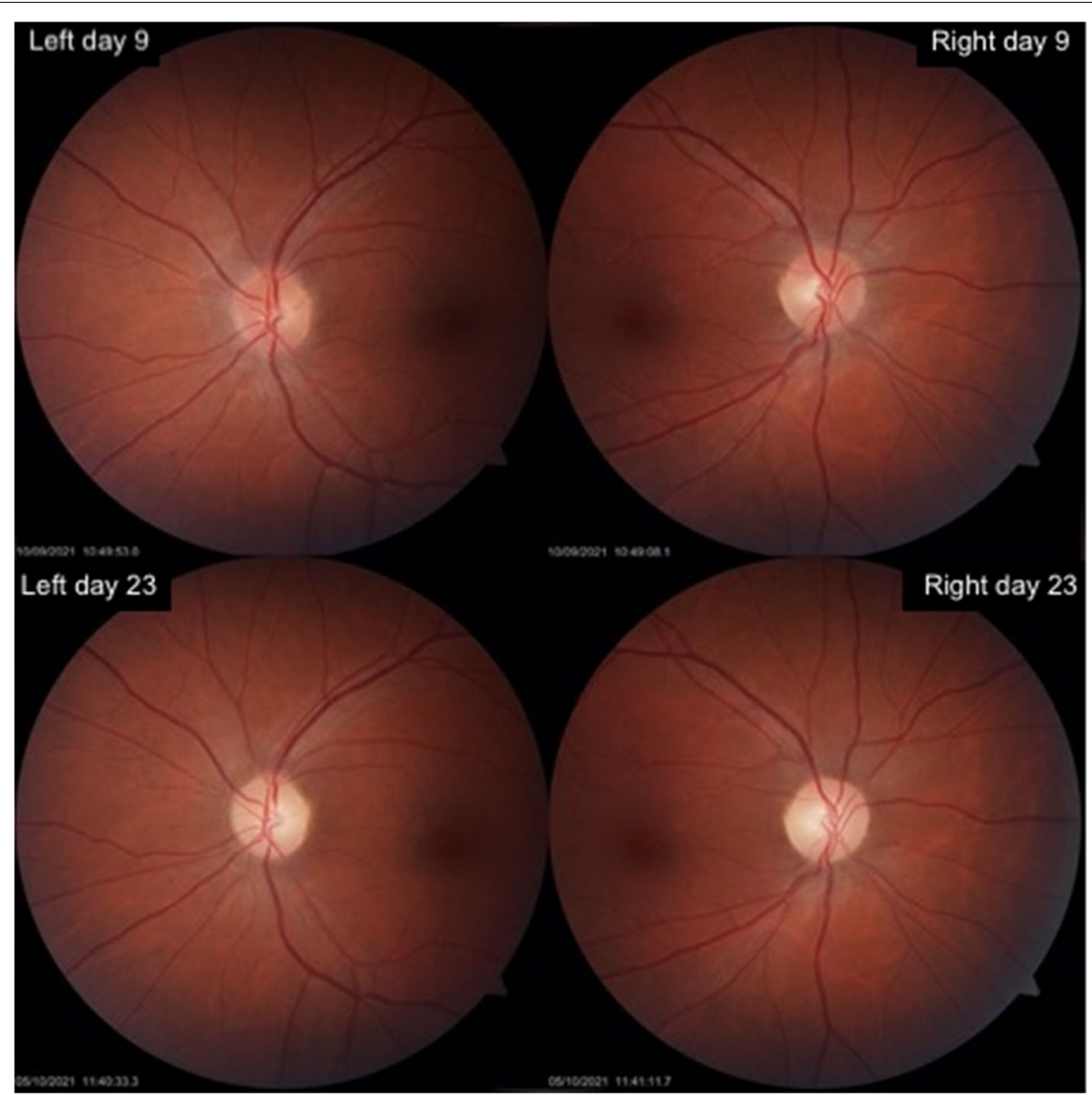

Fig. 2 Photos of the optic nerves of the patient. Left and right optic nerves day 9 after symptom onset did not demonstrate any swelling or atrophy. Day 23 after symptom onset shows pallor bilaterally

testing. Visual field testing of the right eye demonstrated severe depression with an inferior altitudinal defect (Supplementary file 1). Although there was no significant change in retinal nerve fibre layer thickness detectable in the right eye, the left increased by $12 \mu \mathrm{m}$ (Supplementary file 2).

A repeat MRI Brain on day 17 from initial presentation, 2 weeks after the initial MRI showed significant reduction in size of the multiple T2/ FLAIR hyperintense lesions throughout the subcortical white matter. The right frontal centrum semiovale lesion reduced in size to $12 \times 10 \mathrm{~mm}$ with contrast enhancement no longer present (Fig. 1c, d). No interval development of new lesions was evident. However, there was now abnormal signal and enhancement of both optic nerves but more prominent on the left, consistent with optic neuritis. These radiological findings mirrored the results of the visually evoked potentials performed during the first admission.

A further course of 3 doses of $1000 \mathrm{mg}$ daily of intravenous methylprednisone improved her visual symptoms and she was discharged home on $50 \mathrm{mg}$ daily of oral prednisolone with a tapering plan.

23 days after her initial symptoms her clinical picture had significantly improved. Her visual acuity was near baseline: right $6 / 5(20 / 16)$, left 6/6 (20/20) with full Ishihara colour vision bilaterally. Both optic nerves demonstrated pallor (Fig. 2). Her visual field testing was near full on the right and although the left was significantly improved, a dense inferior altitudinal defect remained (Supplementary file 1). Her optical coherence tomography imaging revealed the retinal nerve fibre layer swelling had improved bilaterally (Supplementary file 2) but her ganglion cell layer thickness of the retina was thinned by $15 \mu \mathrm{m}$ in each eye suggesting irreversible optic nerve damage (Supplementary file 3).

Repeat MRI Brain 6 weeks after symptom onset showed further improvement in radiographic appearance of the lesions with no contrast enhancement to suggest active inflammation. Visual evoked potentials at 3 months showed improvement in response bilaterally 


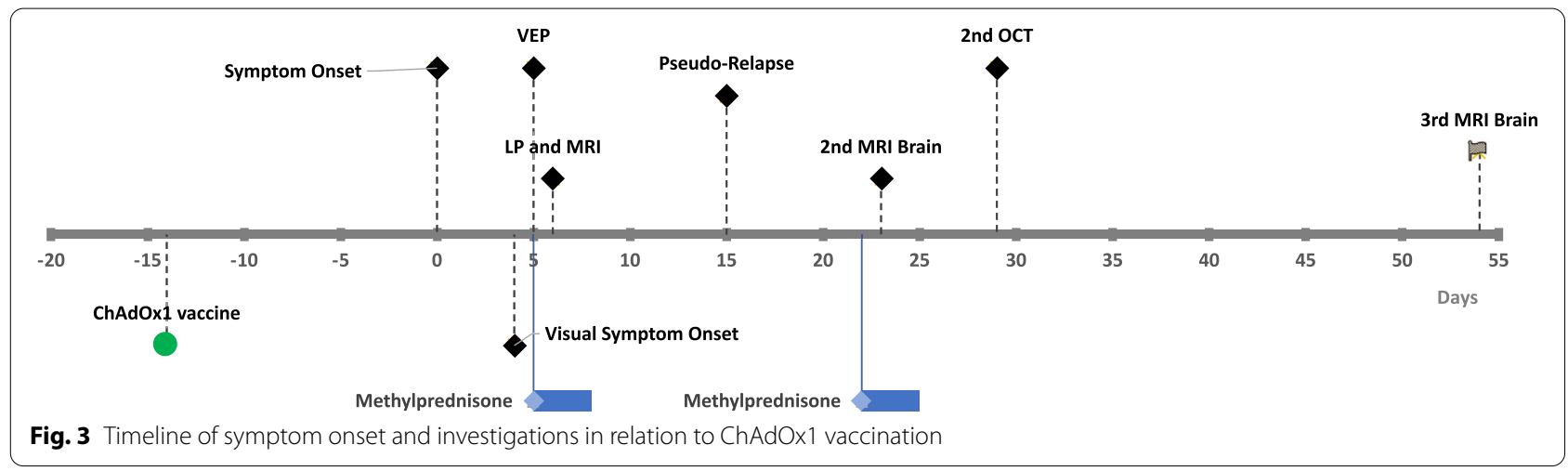

with responses being detectable on the left, where they were previously absent. There were no new symptoms to suggest a clinical relapse, consistent with a monophasic illness. A summary of the timeline of symptoms and investigations is presented in Fig. 3.

\section{Discussion and conclusions}

Our patient presented with clinical, imaging and CSF features consistent with ADEM, with her symptoms starting 14 days following her first dose of ChAdOx1 vaccine. This is the first report of ADEM associated with, or following the ChAdOx1 vaccine, presenting with bilateral optic neuritis.

ADEM is defined as a polysymptomatic event which includes optic neuritis and encephalopathy, that may manifest as behavioural change, confusion, irritability, alteration in consciousness and lethargy [1]. While no diagnostic criteria exists for adults, in children it requires the presence of both encephalopathy and multifocal central nervous system involvement (Table 2) [1]. Optic neuritis is often bilateral and is much more common ( $25 \%)$ as a presenting symptom of both post-viral and post- vaccine ADEM, as in our patient, than in multiple sclerosis where it is relatively rare [12-14]. Evidence of inflammation within the cerebrospinal fluid with pleocytosis and lack of intrathecal oligoclonal band synthesis are more likely in ADEM than in multiple sclerosis, although there is some overlap, as seen in this case [15]. CSF oligoclonal bands were detected in our patient and two other cases of ChAdOx1 associated ADEM [7]. Important differentials in ADEM include multiple sclerosis, neuromyelitis optica spectrum disorder (NMOSD) and myelin oligodendrocyte glycoprotein antibody disease (MOGAD) which we have done our best to exclude [1]. A relapse, as in our case, may occur within 4 weeks of steroid tapering or within 3 months of the sentinel event, but is defined as a part of the monophasic illness [4].

MRI findings suggestive of ADEM include multifocal punctate to large flocculent T2/FLAIR hyperintensities that are often bilateral and asymmetrical, involving peripheral grey and white matter junction. Tumefactive, mass-like lesions are also possible. The brainstem and posterior fossa can be involved, but usually not the callososeptal interface. Enhancement pattern is variablepunctate, ring, incomplete ring, peripheral enhancement have all been reported and absence of enhancement does not exclude the diagnosis [16]. Findings can overlap with those of multiple sclerosis which has predilection for the corpus callosum and periventricular white matter as well as the subcortical U fibres [16]. Active demyelination plaques in multiple sclerosis typically demonstrate incomplete ring enhancement. In our patient the internal punctate foci of enhancement are not typical of an active plaque but is more suggestive of ADEM. This, in combination with absence of lesions in the corpus callosum or lesions involving the subcortical $U$ fibres renders ADEM a more likely radiological differential diagnosis than multiple sclerosis.

Table 2 Definition of monophasic ADEM. From consensus definition for paediatric demyelinating disorders by Krupp et. al [1]

Clinical event with presumed inflammatory or demyelinating cause, with acute of subacute onset that affects multifocal areas of the CNS.

Clinical presentation must be polysymptomatic and must include encephalopathy

Encephalopathy is defined as one or more of the following.

-Behavioural change e.g. confusion, irritability

-Alteration in consciousness e.g. lethargy, coma

Clinical event followed by clinical and/ or radiological improvement with possibility of residual deficits

No history of clinical episodes with features of a prior demyelinating event 
Table 3 Reported cases of ChAdOx1 associated ADEM

\begin{tabular}{|c|c|c|c|c|c|c|c|}
\hline Case & Age/ Sex & $\begin{array}{l}\text { Days post ChAdOx1 } \\
\text { vaccine at symptom } \\
\text { onset }\end{array}$ & $\begin{array}{l}\text { Optic Nerve } \\
\text { involvement }\end{array}$ & $\begin{array}{l}\text { Spinal cord } \\
\text { involvement }\end{array}$ & $\begin{array}{l}\text { CSF } \\
\text { oligoclonal } \\
\text { bands }\end{array}$ & Encephalitis & Treatment \\
\hline Our case & 36, Female & 14 & Yes & No & Yes & Yes (Lethargy) & $\begin{array}{l}\text { Intravenous meth- } \\
\text { ylprednisone, oral } \\
\text { corticosteroids }\end{array}$ \\
\hline Permezal, et al. [6] & 63, Male & 12 & No & Yes & Yes & Yes (Poorly responsive) & $\begin{array}{l}\text { Intravenous } \\
\text { methylprednisone, } \\
\text { plasmapheresis }\end{array}$ \\
\hline Rinaldi et al. [7] & 45, Male & 12 & No & Yes & Yes & No & $\begin{array}{l}\text { Intravenous meth- } \\
\text { ylprednisone, oral } \\
\text { corticosteroids }\end{array}$ \\
\hline Mumoli et al. [8] & 45, Male & 7 & No & Yes & No & No & $\begin{array}{l}\text { Intravenous meth- } \\
\text { ylprednisone }\end{array}$ \\
\hline
\end{tabular}

Specifically for the ChAdOx1 vaccine, there have been approximately 11.6 million doses administered just in Australia as of $20^{\text {th }}$ September 2021 [17]; many more doses have been administered globally. Transverse myelitis, another disorder of central nervous system demyelination, has already been associated with SARS-CoV-2 vaccinations, with at least two cases being reported following ChAdOx1 vaccination [18-20]. Shared antigenic epitopes between a virus or vaccine protein and host myelin protein result in $\mathrm{T}$ and subsequent $\mathrm{B}$-cell activation from antigen presentation is thought to initiate an immune inflammatory reaction and demyelination in both conditions [21].

There have been 3 other cases of ChAdOx1 associated ADEM in the literature (Table 3) [6-8]. Symptom onset occurred between 7-14 days from vaccine administration. Our case is somewhat different from the previous cases of ChAdOx1 associated ADEM - the patient presented with bilateral optic neuritis and there was no evidence of spinal cord involvement, which was a feature of the other reported cases.

While this individual had a family history of multiple sclerosis affecting a first degree relative, there is paucity of data on significance of MS family history in adult onset ADE, and no definite association in the paediatric population has been identified [22, 23]. It remains to be seen in a family history of MS confers increased risk of developing ADEM.

Another interesting aspect of this case is that despite clear initial symptoms suggesting optic nerve involvement, the first MRI did not demonstrate any degree of optic nerve enhancement despite the clearly abnormal VEP, confirming its higher sensitivity than MRI for optic neuritis and its utility still in twenty-first century [24].

Corticosteroids are the mainstay of therapy for ADEM, however plasma exchange or intravenous immunoglobulin may be considered for refractory cases [4]. Complete recovery is the expected course in the majority of postvaccination cases of ADEM [25].

While ADEM does occur following vaccination, ADEM is a vanishingly rare complication following ChAdOx1 vaccine. ADEM should be considered in patients presenting with visual disturbances following ChAdOx1 vaccination, even without other long tract findings. It is imperative as clinicians to be aware and remain vigilant for potential rare complications of vaccinations such as ADEM to improve our understanding of immune system and ADEM. Our report, by highlighting the rarity and reversibility of ADEM, help to emphasise the safety of vaccines that prevent serious illness and death, particularly in a global pandemic.

\section{Abbreviations}

ADEM: Acute disseminated encephalomyelitis.

\section{Supplementary Information}

The online version contains supplementary material available at https://doi. org/10.1186/s12883-022-02575-8.

Additional file 1. Humphrey visual field tests of the left and right eye. Dots represent visual field points tested with normal field. Shaded or black squares denote partially or severely depressed field respectively at this test point. The left eye visual acuity was very poor and the eye deviated outward on September $22^{\text {nd }}$ so could not complete the test. By September $30^{\text {th }}$ the left eye vision had much improved and the now recordable but still depressed visual field is seen. In the right eye, a severe visual field deficit is seen on September $22^{\text {nd }}$ which almost completely resolves by September $30^{\text {th }}$

Additional file 2. Optic coherence tomography of each eye analysing optic nerve retinal nerve fibre layer thickness. Both eyes were mildly thickened on presentation. On relapse the left nerve fibre layer thickened significantly whereas the right eye was largely unchanged. On final review the nerve fibre layer swelling had improved bilaterally.

Additional file 3. Optical coherence tomography of each eye analysing ganglion cell layer thickness at the macula. Both eyes demonstrate sequential thinning of the ganglion cell layer over each visit suggesting irreversible optic nerve damage. 


\section{Acknowledgements \\ Not applicable}

\section{Authors' contributions}

SAN: Conception of work, analysed and interpreted neurological findings, drafted manuscript. AF: Analysed and interpreted ophthalmological findings, drafted ophthalmological aspects of manuscript. JL: Analysed and interpreted ophthalmological findings, drafted ophthalmological aspects of manuscript. $\mathrm{RL}$ : Analysed and interpreted radiological findings, drafted radiological aspects of manuscript. YH: Conception of work, analysed and interpreted neurological findings, drafted manuscript. RH: Analysed and interpreted neurological findings, drafted manuscript. All authors read and approved the final manuscript.

\section{Funding}

Not applicable.

\section{Availability of data and materials}

Not applicable.

\section{Declarations}

\section{Ethics approval and consent to participate}

Not applicable.

\section{Consent for publication}

Patient written consent obtained. Can be provided if required.

\section{Competing interests}

The authors did not receive support from any organization for the submitted work. There are no competing interests to declare.

\section{Author details}

${ }^{1}$ Department of Neurology, Gosford Hospital, Gosford, NSW, Australia. ${ }^{2}$ The University of Sydney, Save Sight Institute, Sydney, NSW, Australia. ${ }^{3}$ Sydney Eye Surgeons, Sydney, NSW, Australia. ${ }^{4}$ Department of Radiology, Gosford Hospital, Gosford, NSW, Australia. ${ }^{5}$ Brain and Mind Centre, The University of Sydney and Gosford Hospital, Gosford, NSW, Australia. ${ }^{6}$ The University of Sydney, Sydney, NSW, Australia.

Received: 8 November 2021 Accepted: 28 January 2022 Published online: 12 February 2022

\section{References}

1. Krupp LB, Banwell B, Tenembaum S. Consensus definitions proposed for pediatric multiple sclerosis and related disorders. Neurology. 2007;68(16 suppl 2):S7.

2. Cole J, Evans E, Mwangi M, Mar S. Acute Disseminated Encephalomyelitis in Children: An Updated Review Based on Current Diagnostic Criteria. Pediatr Neurol. 2019;100:26-34.

3. Otallah S. Acute disseminated encephalomyelitis in children and adults: A focused review emphasizing new developments. Mult Scler J. 2021;27(8):1153-60.

4. Tenembaum S, Chitnis T, Ness J, Hahn JS. Acute disseminated encephalomyelitis. Neurology. 2007;68(16 suppl 2):S23.

5. Zelada-Ríos L, Pacheco-Barrios K, Galecio-Castillo M, Yamunaqué-Chunga C, Álvarez-Toledo K, Otiniano-Sifuentes R. Acute disseminated encephalomyelitis and COVID-19: A systematic synthesis of worldwide cases. J Neuroimmunol. 2021;359:577674.

6. F Permezel B Borojevic S Lau HH de Boer 2021 Acute disseminated encephalomyelitis (ADEM) following recent Oxford/AstraZeneca COVID19 vaccination Forensic Sci Med Pathol. Online ahead of print.

7. Rinaldi V, Bellucci G, Romano A, Bozzao A, Salvetti M. ADEM after ChAdOx1 nCoV-19 vaccine: A case report. Multiple Sclerosis Journal. 2021:13524585211040222. Online ahead of print

8. Mumoli L, Vescio V, Pirritano D, Russo E, Bosco D. ADEM anti-MOG antibody-positive after SARS-CoV2 vaccination. Neurol Sci. 2021;43(2):763-6.
9. Vogrig A, Janes F, Gigli GL, Curcio F, Negro ID, D'Agostini S, et al. Acute disseminated encephalomyelitis after SARS-CoV-2 vaccination. Clin Neurol Neurosurg. 2021;208:106839.

10. Cao L, Ren L. Acute disseminated encephalomyelitis after severe acute respiratory syndrome coronavirus 2 vaccination: a case report. Acta Neurol Belg. 2021:1-3. Online ahead of print.

11. Ozgen Kenangil G, Ari BC, Guler C, Demir MK. Acute disseminated encephalomyelitis-like presentation after an inactivated coronavirus vaccine. Acta Neurol Belg. 2021;121(4):1089-91.

12. Bangsgaard R, Larsen VA, Milea D. Isolated bilateral optic neuritis in acute disseminated encephalomyelitis. Acta Ophthalmol Scand. 2006:84(6):815-7.

13. Burman J, Raininko R, Fagius J. Bilateral and recurrent optic neuritis in multiple sclerosis. Acta Neurol Scand. 2011;123(3):207-10.

14. Lapphra K, Huh L, Scheifele DW. Adverse neurologic reactions after both doses of pandemic H1N1 influenza vaccine with optic neuritis and demyelination. Pediatr Infect Dis J. 2011;30(1):84-6.

15. Dale RC, de Sousa C, Chong WK, Cox TCS, Harding B, Neville BGR. Acute disseminated encephalomyelitis, multiphasic disseminated encephalomyelitis and multiple sclerosis in children. Brain. 2000;123(12):2407-22.

16. Osborn AG, Salzman KL, Barkovich AJ. Diagnostic Imaging: Brain. Salt Lake City: Amirsys; 2010.

17. Therapeutic Goods Administration DoH. COVID-19 vaccine weekly safety report - 30-09-2021 Australia Australian Government 2021 [updated 30/09/21. Available from: https://www.tga.gov.au/periodic/covid-19vaccine-weekly-safety-report-30-09-2021.

18. Voysey M, Clemens SAC, Madhi SA, Weckx LY, Folegatti PM, Aley PK, et al. Safety and efficacy of the ChAdOx1 nCoV-19 vaccine (AZD1222) against SARS-CoV-2: an interim analysis of four randomised controlled trials in Brazil, South Africa, and the UK. The Lancet. 2021;397(10269):99-111.

19. Román GC, Gracia F, Torres A, Palacios A, Gracia K, Harris D. Acute Transverse Myelitis (ATM):Clinical Review of 43 Patients With COVID19-Associated ATM and 3 Post-Vaccination ATM Serious Adverse Events With the ChAdOx1 nCoV-19 Vaccine (AZD1222). Front Immunol. 2021;12(879):653786.

20. Malhotra HS, Gupta P, Prabhu V, Kumar Garg R, Dandu H, Agarwal V. COVID-19 vaccination-associated myelitis. QJM: Int J Med. 2021:114(8):591-3.

21. Menge T, Kieseier BC, Nessler S, Hemmer B, Hartung HP, Stüve O. Acute disseminated encephalomyelitis: an acute hit against the brain. Curr Opin Neurol. 2007;20(3):247-54.

22. Pohl D, Hennemuth I, von Kries R, Hanefeld F. Paediatric multiple sclerosis and acute disseminated encephalomyelitis in Germany: results of a nationwide survey. Eur J Pediatr. 2007;166(5):405-12.

23. Alper G, Heyman R, Wang L. Multiple sclerosis and acute disseminated encephalomyelitis diagnosed in children after long-term follow-up: comparison of presenting features. Dev Med Child Neurol. 2009;51(6):480-6.

24. Miller DH, Newton MR, van der Poel JC, du Boulay EPGH, Halliday AM, Kendall BE, et al. Magnetic resonance imaging of the optic nerve in optic neuritis. Neurology. 1988;38(2):175.

25. Huynh W, Cordato DJ, Kehdi E, Masters LT, Dedousis C. Post-vaccination encephalomyelitis: Literature review and illustrative case. J Clin Neurosci. 2008;15(12):1315-22

\section{Publisher's Note}

Springer Nature remains neutral with regard to jurisdictional claims in published maps and institutional affiliations. 